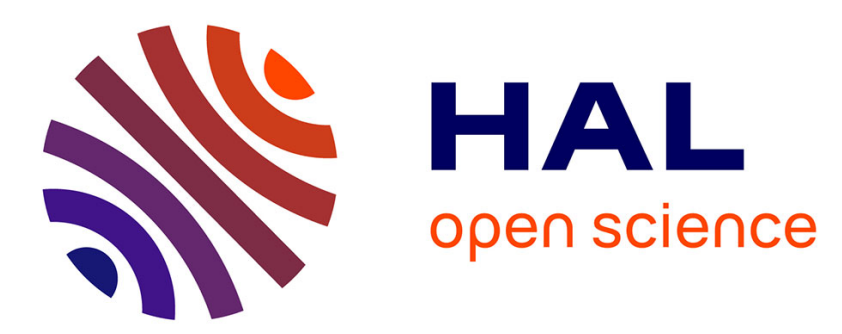

\title{
Evidence for the formation of two phases during the growth of SrTiO3 on silicon
}

\author{
G. Niu, J. Penuelas, L. Largeau, B. Vilquin, J.-L. Maurice, C. Botella, G. \\ Hollinger, G. Saint-Girons
}

\section{- To cite this version:}

G. Niu, J. Penuelas, L. Largeau, B. Vilquin, J.-L. Maurice, et al.. Evidence for the formation of two phases during the growth of SrTiO3 on silicon. Physical Review B: Condensed Matter and Materials Physics (1998-2015), 2011, 83, pp.054105. 10.1103/PhysRevB.83.054105 . hal-00601296

\section{HAL Id: hal-00601296 \\ https://hal-polytechnique.archives-ouvertes.fr/hal-00601296}

Submitted on 17 Jun 2011

HAL is a multi-disciplinary open access archive for the deposit and dissemination of scientific research documents, whether they are published or not. The documents may come from teaching and research institutions in France or abroad, or from public or private research centers.
L'archive ouverte pluridisciplinaire HAL, est destinée au dépôt et à la diffusion de documents scientifiques de niveau recherche, publiés ou non, émanant des établissements d'enseignement et de recherche français ou étrangers, des laboratoires publics ou privés. 


\title{
Evidence for the formation of two phases during the growth of $\mathrm{SrTiO}_{3}$ on silicon
}

\author{
G. Niu, ${ }^{1}$ J. Penuelas, ${ }^{1}$ L. Largeau, ${ }^{2}$ B. Vilquin, ${ }^{1}$ J. L. Maurice, ${ }^{3}$ C. Botella, ${ }^{1}$ G. Hollinger, ${ }^{1}$ and G. Saint-Girons ${ }^{1, *}$ \\ ${ }^{1}$ Université de Lyon, Institut des Nanotechnologies de Lyon (UMR5270/CNRS), Ecole Centrale de Lyon, 36 avenue Guy de Collongue, \\ F-69134 Ecully cedex, France \\ ${ }^{2}$ LPN-UPR20/CNRS, Route de Nozay, F-91460 Marcoussis, France \\ ${ }^{3}$ LPICM CNRS/Ecole Polytechnique, F-91128 Palaiseau cedex, France
}

(Received 8 October 2010; published 15 February 2011)

\begin{abstract}
Epitaxial $\mathrm{SrTiO}_{3}(\mathrm{STO}) / \mathrm{Si}$ templates open a unique opportunity for the integration of ferroelectric oxides, such as $\mathrm{BaTiO}_{3}$ on silicon and for the realization of new devices exploiting ferroelectricity. STO itself has been shown as ferroelectric at room temperature when deposited in thin layers on Si, while bulk STO is tetragonal and, thus, ferroelectric below $105 \mathrm{~K}$. Here, we demonstrate the coexistence, at room temperature, of strained cubic and tetragonal phases in thin STO/Si layers. The tetragonal STO phase presents a pronounced tetragonality for thicknesses up to $24 \mathrm{ML}$. Above this thickness, the strained cubic STO phase starts relaxing while the tetragonal STO phase progressively transits to cubic STO. The origin of the simultaneous formation of these two phases is analyzed and is attributed to oxygen segregation at the early stages of the growth.
\end{abstract}

DOI: 10.1103/PhysRevB.83.054105

PACS number(s): 77.55.fp, 68.55.aj, 61.05.cp

\section{INTRODUCTION}

So-called functional oxides crystallizing in the perovskite structure present a wide range of electronic properties (ferromagnetism, ferroelectricity, piezoelectricity, thermoelectricity, supraconductivity,...) and are actively studied for the realization of high-performance devices (switches, radiofrequency filters, ferroelectric transistors and memories, spintronic devices, mechanic energy conversion systems, micro- and nanoelectromechanical systems, ....). ${ }^{1-9}$ For this purpose, crystalline perovskite layers and heterostructures are most often grown on commercially available $\mathrm{SrTiO}_{3}$ (STO) substrates. However, the structural quality, limited size, and cost of these substrates make them inappropriate for industrial applications. Since the pioneering work of McKee et al., ${ }^{10,11}$ several groups have focused their research on the epitaxial growth of STO thin films on silicon. ${ }^{12-15}$ High-quality $\mathrm{STO} / \mathrm{Si}$ templates allow for envisaging the direct growth of perovskite-oxide-based heterostructures on $\mathrm{Si}$ platforms and could be used for the monolithic integration of III-V semiconductors on silicon due to the unique properties of the semiconductor/STO heterointerfaces. ${ }^{16-18}$ The STO/Si epitaxial system is characterized by a strong crystallographic heterogeneity (STO crystallizes in the perovskite Pm-3m lattice and presents a bulk lattice parameter of $3.905 \AA$ at room temperature, while $\mathrm{Si}$ has a diamond Ia-3 lattice structure and a room temperature lattice parameter of $5.431 \AA$ ). During the molecular beam epitaxy (MBE) of STO on Si, the oxide aligns its 100 in-plane directions with the 110 in-plane directions of the $\mathrm{Si}$ surface, leading to a reduced effective misfit of $1.7 \%$. Moreover, particular care has to be taken to avoid silicate or $\mathrm{SiO}_{2}$ formation at the $\mathrm{STO} / \mathrm{Si}$ interface, even using the McKee Si passivation procedure. Among the various methods used to circumvent this difficulty, ${ }^{5,19,20}$ the so-called kinetically controlled STO growth ${ }^{15,21}$ (KCSD process) gives the most promising results. It consists of depositing a few STO monolayers at low temperature $\left(T<300{ }^{\circ} \mathrm{C}\right)$ and in annealing the material under very low oxygen pressure at $550{ }^{\circ} \mathrm{C}$. This procedure is repeated several times until obtaining the desired thickness. During the low-temperature STO deposition step, amorphous and crystalline STO coexist during 1 to $2 \mathrm{ML}^{22}$ The amorphous STO part recrystallizes during annealing. This sequential growth process significantly complicates the understanding of the interaction between growing material and substrate due to recrystallization and because annealing leads to oxygen evaporation, which may impact STO structural properties.

Warusawithana et al. have recently reported room temperature ferroelectricity of thin STO/Si layers grown using the KCSD process, ${ }^{23}$ opening promising scientific and applicative perspectives. A strong correlation between STO crystallographic coherency with respect to $\mathrm{Si}$ and ferroelectricity of the oxide layer has been observed, bringing the authors to the conclusion that epitaxial strain is at the origin of the ferroelectric behavior of the thin layers. Besides this, experimental studies supported by density functional theory calculations ${ }^{24,25}$ suggest that charged defects, such as interface oxygen vacancies, possibly combined with $-\mathrm{OH}$ adsorbates on the oxide surface, may promote ferroelectricity by efficiently screening the depolarization field in thin STO/Si films. In this paper, we produce concluding experimental elements concerning the ferroelectric behavior of thin epitaxial STO/Si layers. The structural properties of thin STO/Si layers grown as in the low-temperature step of the KCSD method are analyzed in order to avoid additional complexity related to annealing. This paper is based on the analysis of $\mathrm{x}$-ray diffraction (XRD) data, transmission electron microscopy (TEM) measurements, and reflection high-energy electron diffraction (RHEED) experiments.

\section{STO GROWTH CONDITIONS}

The STO thin films considered in the following were deposited on $p$-doped silicon substrates in a RIBER 2300 MBE reactor equipped for oxide growth. Si substrates were prepared as detailed in Ref. 22. Sr and Ti were evaporated using Knudsen effusion cells, while purified oxygen was introduced under its molecular $\mathrm{O}_{2}$ form via a differentially 
pumped prechamber allowing for precise control of the $\mathrm{O}_{2}$ partial pressure. Sr passivation of the Si surface was carried out using the McKee procedure. ${ }^{10}$ The resulting $\mathrm{SrSi}_{2}$ surface reconstruction was then first exposed to an $\mathrm{O}_{2}$ partial pressure of $5 \times 10^{-8}$ Torr during $1 \mathrm{~min}$ at $360{ }^{\circ} \mathrm{C}$ before starting STO growth under the same $\mathrm{O}_{2}$ pressure and temperature conditions, at a growth rate of $0.019 \mathrm{ML} \mathrm{s}^{-1}$ (monitored using RHEED intensity oscillations). After 1 to $2 \mathrm{ML}$, the $\mathrm{O}_{2}$ partial pressure was ramped up to $5 \times 10^{-6}$ Torr and stabilized at this value during the rest of the growth. Some 10-, 14-, 24-,
42-, and 118-ML-thick STO/Si layers were fabricated using the growth conditions described earlier. These samples were characterized by TEM and by XRD. For XRD experiments, a Rigaku Smartlab diffractometer equipped with a 9-kW rotating anode and an in-plane arm was used. For out-of-plane experiments, the $\mathrm{Cu}-\mathrm{K} \alpha_{1}$ radiation of the x-ray source was monochromatized using a two-bounce Ge220 monochromator, leading to an $\omega$ resolution of $0.011^{\circ}$. In-plane experiments were carried out using $0.5^{\circ}$ Soller slits placed on incident and diffracted beams, leading to a $\phi$ resolution better than
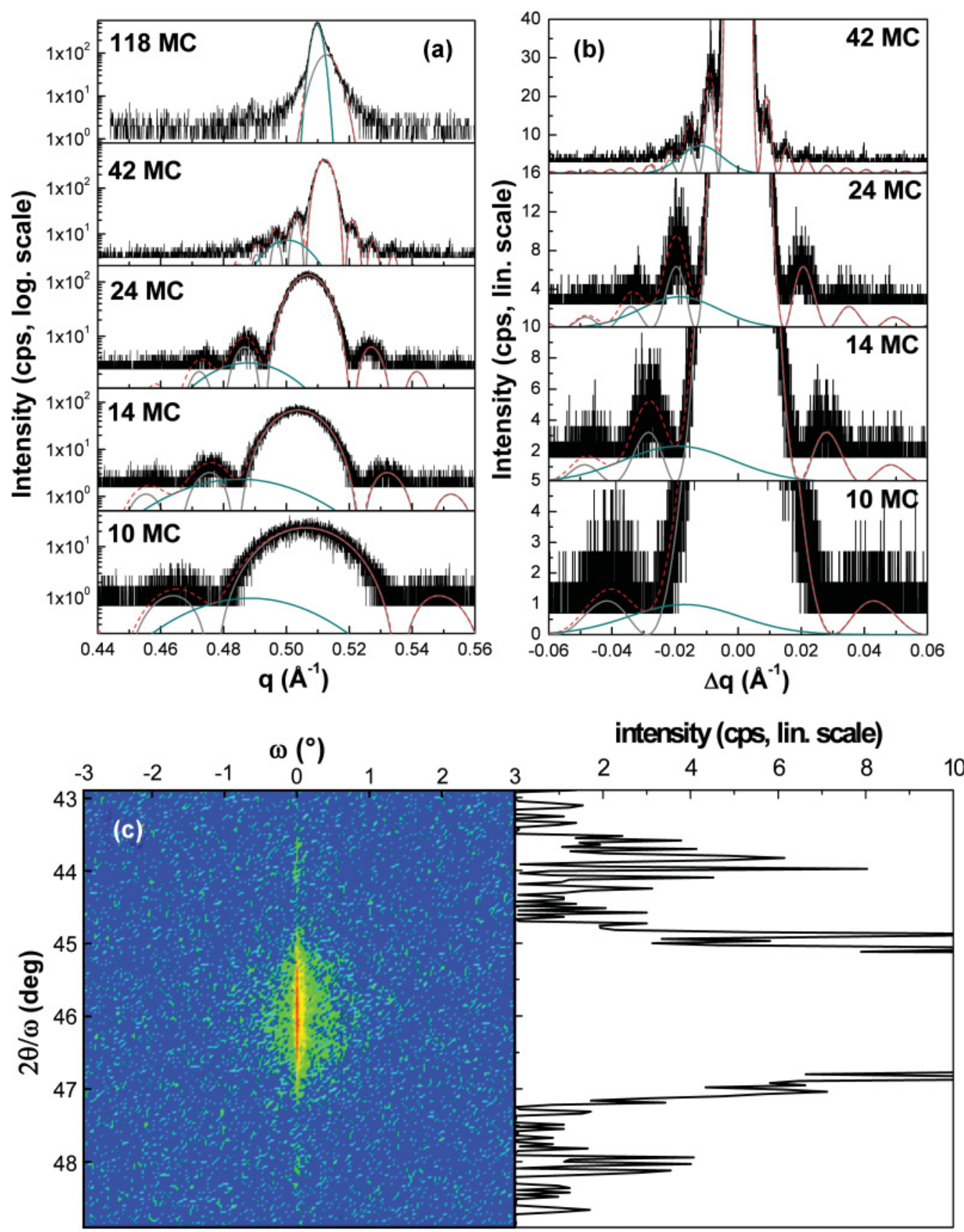

FIG. 1. (Color online) (a) $2 \theta-\omega$ scans of the STO 002 reflection for 10-, 14-, 24-, 42-, and 118-ML-thick STO/Si layers. (Gray, dark-cyan, and red-dashed lines) Fit of the peaks. $q=1 / d$ is the interatomic distance in the growth direction. (b) Zooms in linear scale on the Pendellösung fringes of the spectra of (a). $\Delta q$ is the offset with respect to the center of the cardinal sinus contribution. (c) Reciprocal space map of the STO 002 reflection and $2 \theta-\omega$ profile of the map for $\omega=0$. 
$0.41^{\circ}, \phi$ being the azimuthal rotation angle (rotation around the substrate normal).

\section{TWO-PHASED STO, STRAIN RELAXATION}

The XRD out of plane $2 \theta-\omega$ scans recorded around the STO 002 reflection for the five samples are displayed in Fig. 1(a) (in reciprocal space units: $q=1 / d$ where $d$ is the distance in the real space along the growth axis). The spectra of the four thinnest samples $(10,14,24$, and $42 \mathrm{ML})$ present Pendellösung fringes attesting for the good crystallinity and flatness of the STO layers. The main diffraction peak progressively shifts toward large $q$ values increasing the STO thickness beyond $14 \mathrm{ML}$, indicating a decrease of the STO out-of-plane lattice parameter. At $118 \mathrm{ML}$, Pendellösung fringes are no longer detected due to a degradation of the STO quality associated with plastic relaxation (see the following). Interestingly, the Pendellösung fringes are not symmetric for the 10-, 14-, 24-, and 42-ML samples. The XRD spectra cannot be fitted using a simple cardinal sinus function, and a shoulder peak at low $q$ value must be added, as shown in Fig. 1(b). Good agreement between experimental data and fitted curves is obtained by using a sum of a cardinal sinus (for the main diffraction peak and the Pendellösung fringes) and a Gaussian function (for the low $q$ shoulder). Such a dissymmetry of the diffraction spectrum can result from geometric effects (strong mosaicity of the STO layer, possible misalignment between the STO layer, and the Si substrate). To test this hypothesis, a reciprocal space map of the STO 002 reflection of the 24-ML-thick sample was recorded using a Ge 200 two-bounce analyzer on the diffracted beam [Fig. 1(c)]. This allows for strongly reducing the detection acceptance angle, thus, removing possible parasitic diffraction due to geometric effects. The profile extracted for the reciprocal space map of Fig. 1(c) at $\omega=0$ is also dissymmetric, indicating that this dissymmetry is related to the presence in the layers of two different STO domains having two different out-ofplane parameters (designated as $\mathrm{c}$ in the following). The 002 STO peak of the 118-ML-thick sample can also clearly be decomposed into two components, as shown in Fig. 1(a).

In-plane diffraction was used to record the $2 \theta \chi-\phi$ spectra corresponding to the 110 STO reflections. The $2 \theta \chi$ angle in the in-plane configuration is equivalent to the $2 \theta$ angle in the out-of-plane configuration so that in-plane $2 \theta \chi-\phi$ scans are equivalent to $2 \theta-\omega$ out-of-plane scans. The 110 STO reflections are particularly interesting because $100 \mathrm{Si}$ reflections, which are theoretically located in the same angular region, are forbidden due to diffraction selection rules. Thus, diffraction from STO only is collected around these reflections. A deconvolution into two Gaussian peaks was required to fit these $2 \theta \chi-\phi$ scans, as shown in Fig. 2. The bimodal character of the diffraction peaks is clear for the 10-, 14-, and 24-ML samples and becomes less clear for larger thicknesses. However, even for the 42- and 118-ML-thick layers, a better fit was obtained using two Gaussian peaks rather than one.

This analysis indicates that the STO layers contain two STO domains having two different in-plane lattice parameters (designated as a in the following) and two different outof-plane lattice parameters c. The lattice parameter values extracted from in- and out-of-plane XRD experiments are

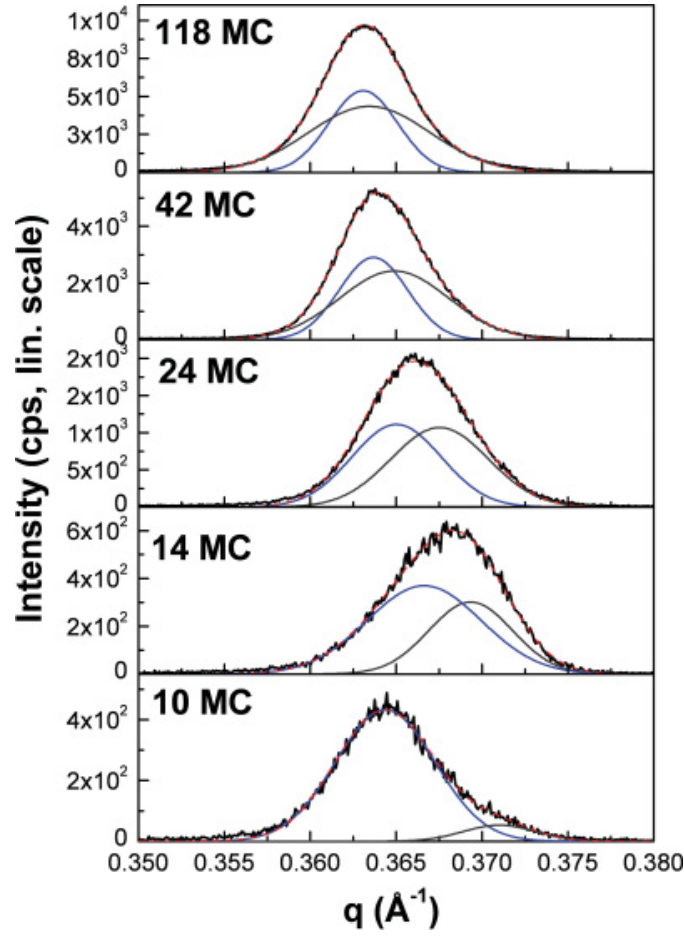

FIG. 2. (Color online) In-plane $2 \theta \chi-\phi$ scans of the STO 110 reflections for 10-, 14-, 24-, 42-, and 118-ML-thick STO/Si layers. (Dark-gray, blue, and red-dashed lines) Fit of the peaks. $q=1 / d$ is the interatomic distance in the growth plane.

plotted in Fig. 3. In this figure, the red dashed-dotted lines represent the values calculated for in- and out-of-plane lattice parameters of fully strained and fully relaxed (bulk) cubic Pm-3m STO. For this calculation, STO elastic constants

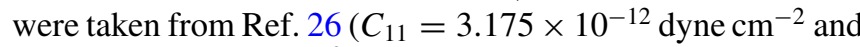

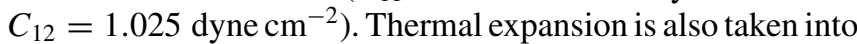
account in this calculation: The thermal expansion coefficient of STO $\left(\alpha_{\text {STO }}=9 \times 10^{-6}{ }^{\circ} \mathrm{C}^{-1}\right.$, Ref. 27) is much larger than

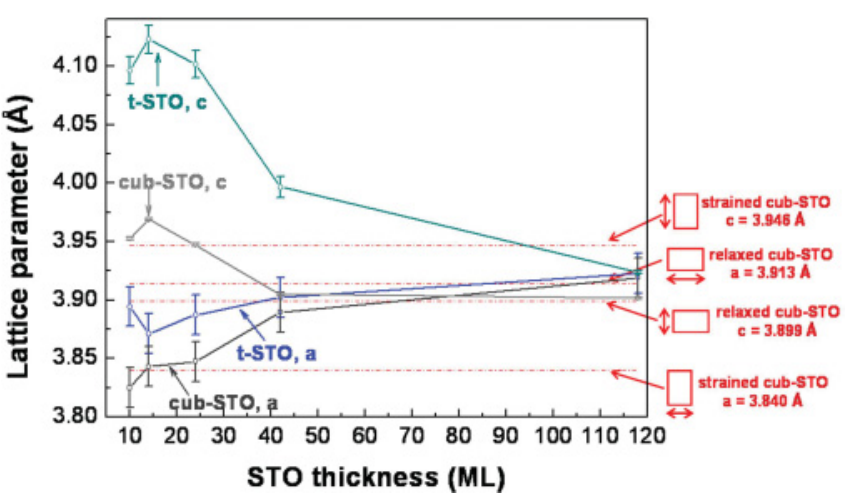

FIG. 3. (Color online) Evolution of the in-plane a and out-ofplane c STO lattice parameters as a function of the STO thickness in monolayers, as deduced from XRD experiments. (Gray and dark lines) the evolution of $\mathrm{c}$ and a, respectively, for the cubic STO phase and (dark-cyan and blue lines) the evolution of $\mathrm{c}$ and a, respectively, for the tetragonal t-STO phase. (Red-dashed lines) Calculated values for $\mathrm{c}$ and a for fully strained and fully relaxed cubic STO, taking into account the influence of the Si substrate on the thermal expansion of STO during the temperature ramping-down step after growth. 
that of $\operatorname{Si}\left(\alpha_{S i}=2.5 \times 10^{-6}{ }^{\circ} \mathrm{C}^{-1}\right.$, Ref. 27). As a consequence, thin STO layers grown on Si substrates undergo additional thermal deformation after epitaxy, when the temperature is ramped from the growth temperature $\left(360{ }^{\circ} \mathrm{C}\right)$ down to the ambient temperature $\left(20{ }^{\circ} \mathrm{C}\right)$. As described in Ref. 28 for the GaAs/Si system, the in-plane lattice parameter of STO is submitted to the $\mathrm{Si}$ thermal expansion coefficient, while the effective STO out-of-plane thermal expansion coefficient depends on the thermal expansion coefficients of Si and STO as well as on STO elastic constants and rates $1.315 \times 10^{-5}{ }^{\circ} \mathrm{C}^{-1}$. This anisotropic contraction leads to built-in tensile strain at room temperature in STO thin layers relaxed on Si at growth temperature (for such relaxed layers, a is larger than $\mathrm{c}$ ). The dark- and light-gray experimental curves in Fig. 3 labeled cub-STO a and cub-STO c, respectively, correspond to the same STO domain: For thicknesses lower than $24 \mathrm{ML}$, the corresponding experimental values for a and c correspond to the calculated values for standard STO crystallizing in the cubic $\mathrm{Pm}-3 \mathrm{~m}$ perovskite structure and fully strained on the $\mathrm{Si}$ substrate. For this STO phase, designated as cub-STO in the following, plastic relaxation starts between 24 and $42 \mathrm{ML}$, leading to a decrease of $\mathrm{c}$ and an increase of a. For the 118-ML-thick layer, the experimental values of a and $\mathrm{c}$ match that calculated for bulk cubic Pm-3m STO (on the condition that differential thermal expansion is taken into account), showing that the cub-STO phase is fully plastically relaxed for this thickness. According to these experimental results, the critical thickness for plastic relaxation of STO on Si is between 24 and 42 ML.

The remaining set of lattice parameters (blue and dark-cyan curves in Fig. 3 labeled t-STO a and t-STO c, respectively) correspond to a second STO phase, designated as t-STO in the following. Obviously, this t-STO phase does not correspond to cubic Pm-3m STO: The associated c values for the 10-, 14-, and 24-ML-thick STO layers are around 4.1 $\AA$, which are much larger than the expected c value for cubic STO even strained on $\mathrm{Si}$, and the associated a values neither match that expected for cubic STO strained on Si, nor that expected for bulk cubic Pm-3m STO. It corresponds to a tetragonal STO phase, the origin of which will be discussed in further detail in Sec. IV. The tetragonality (c/a-1) as a function of the deposited STO thickness and the evolution of $\mathrm{c}$ as a function of a are plotted for both cub-STO and t-STO phases in Fig. 4. For the cub-STO phase, the tetragonality corresponds to that expected for cubic Pm-3m STO strained on Si for thicknesses below $24 \mathrm{ML}$ and tends toward that expected for fully relaxed STO for larger thicknesses. Moreover, the evolution of $c$ as a function of a matches that expected from linear elasticity for cubic Pm-3m STO [black dashed-dotted line in Fig. 4(b)]. For the t-STO phase, the tetragonality is around 0.06 for thicknesses below $24 \mathrm{ML}$ and tends toward that expected for fully relaxed cubic STO for larger thicknesses. For this t-STO phase, the evolution of $\mathrm{c}$ as a function of a presents two regimes: For thicknesses below $24 \mathrm{ML}$, the experimental points are aligned along a line that can be easily fitted [dark-gray dashed-dotted line in Fig. 4(b)] using a Poisson-type relationship of the form $c=a_{b}-2 \times \frac{C_{12}}{C_{11}} \times\left(a+a_{b}\right)$, where $a_{b}, C_{12}$, and $C_{11}$ are the bulk lattice parameter and the elastic constants of the t-STO phase, respectively. The fit in Fig. 4(b) leads to $a_{b} \sim 4.0 \AA$ and $C_{12} / C_{11} \sim 0.46$, which strongly differs from the values
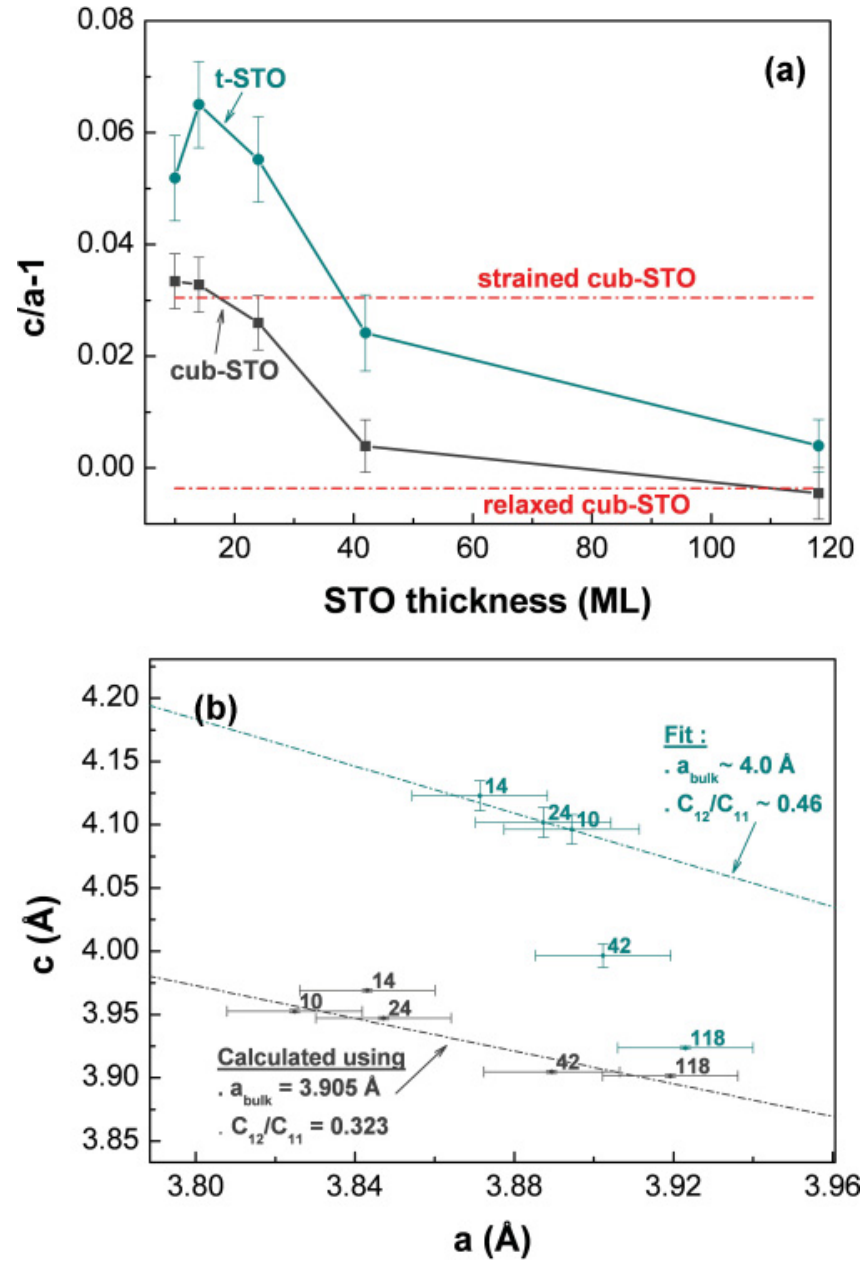

FIG. 4. (Color online) (a) Evolution of the tetragonality (c/a-1) as a function of the STO thickness for the cub-STO phase (dark gray) and the t-STO phase (dark cyan). (Red-dashed lines) Calculated tetragonalities for fully strained and fully relaxed cubic STO, taking into account the influence of the Si substrate on the thermal expansion of STO during the temperature ramping-down step after growth. (b) Evolution of c (out-of-plane lattice parameter) as a function of a (in-plane lattice parameter) as a function of the STO thickness for the cub-STO phase (dark gray) and the t-STO phase (dark cyan). (Dark-gray dashed-dotted line) Elasticity theory prediction for cubic STO (elastic parameters indicated on the graph). (Dark-cyan dashed-dotted line) Fit on the three smallest thicknesses for the t-STO phase (fit parameters indicated on the graph).

for cubic Pm-3m STO $\left(a_{b}=3.905 \AA\right.$ and $\left.C_{12} / C_{11}=0.323\right)$. Interestingly, for the 42- and 118-ML-thick STO layers, the evolution of $\mathrm{c}$ as a function of a for the t-STO phase deviates from that predicted by Poisson relationship and tends toward that measured for the cub-STO phase. This evolution, as well as that of the tetragonality, suggests that the t-STO phase undergoes a progressive tetragonal to cubic phase transition for deposited thicknesses larger than $24 \mathrm{ML}$, which corresponds to the thickness above which the plastic relaxation of the cub-STO phase starts.

In order to further demonstrate the experimental results detailed earlier precisely, the 10-ML-thick STO/Si sample was studied with high-resolution TEM (Fig. 5). A high-resolution 

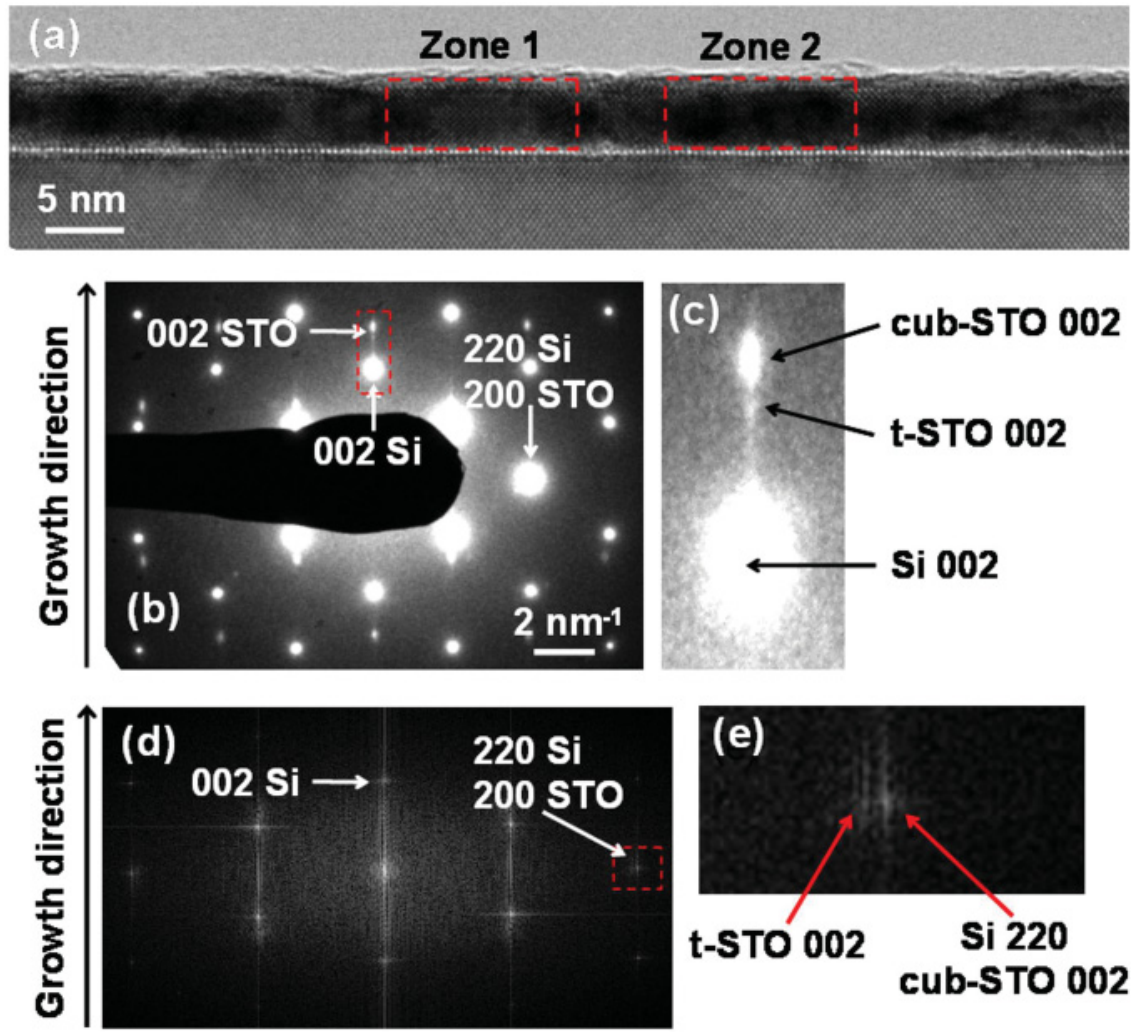

FIG. 5. (Color online) (a) Highresolution TEM cross-sectional view of the 10-ML-thick STO/Si layer. (b) Corresponding electronic diffraction pattern. (c) Zoom around $002 \mathrm{Si}$ and $002 \mathrm{STO}$ out-of-plane reflections [squared zone in (b)]. (d) Fourier-transform of the image of (a) and (e) zoom around the 002 in-plane reflections [squared zone in (d)]. cross-sectional view of this sample is displayed in Fig. 5(a). The STO layer is flat, and its interface with Si is abrupt at the atomic scale. The image contrast in the STO region presents clear heterogeneities, attesting for slight crystallographic disorder. More precisely, well-defined bright zones [as zone 1 in Fig. 5(a)] coexist with darker zones (as zone 2). The electron-diffraction pattern corresponding to the image of Fig. 5(a) is displayed in Fig. 5(b). In the in-plane (horizontal) direction, the Si 220 and STO 200 diffraction spots are superimposed, as expected from the indirect epitaxial relationship between STO and $\mathrm{Si}$. In the growth (vertical) direction, the STO 002 diffraction spot appears clearly. Interestingly, this diffraction spot is doubled, indicating that the STO presents two distinct c parameters. The values of these c parameters, as extracted from the diffraction pattern are $3.95 \pm 3 \times 10^{-2} \AA$ and $4.10 \pm 2 \times 10^{-2} \AA$ for the bright external spot and the less bright internal spot, respectively. These values match that measured by $\mathrm{x}$-ray diffraction for the $\mathrm{c}$ parameters of the strained cub-STO and the t-STO phases, respectively, in the 10-ML-thick sample. The Fourier transform of the image of Fig. 5(a) is displayed in Fig. 5(d). In this Fourier-transform image, the in-plane STO 200 diffraction spot is no longer masked by the Si 220 diffraction spot, and both reflections can be distinguished, as shown by the zoom in the corresponding region [Fig. 5(e)]. The two diffraction spots correspond to a values of $3.845 \pm 8 \times 10^{-3} \AA$ and $3.899 \pm 8 \times 10^{-3} \AA$. The smallest of these values corresponds to the Si 110 interatomic distance and to the a value for strained cub-STO, while the largest corresponds to the a value measured by XRD for the t-STO phase. Thus, this TEM analysis confirms the presence of the two STO phases in the 10-ML-thick samples and confirms the a and $\mathrm{c}$ values measured by XRD.

\section{FORMATION OF THE TWO STO PHASES}

Further information can be extracted from the analysis of the TEM image of Fig. 5(a): The Fourier-transform images corresponding to zones 1 and 2 are displayed in Fig. 6. The horizontal line profiles recorded along the $\langle 200\rangle$ axis in these Fourier-transform images are compared in this figure. This graph shows that STO does not have the same in-plane lattice parameter in zones 1 and 2. In zone 1 [bright zone in Fig. 5(a)], the a value extracted from the Fourier-transform image of Fig. 6 is $3.85 \pm 1.5 \times 10^{-2} \AA$, which is close to that expected for STO strained on Si (3.840 $\AA$ ). In zone 2 [darker zone in Fig. 5(b)], the a value for STO is $3.90 \pm 1.5 \times 10^{-2} \AA$, very close to that measured by XRD for the t-STO in-plane lattice parameter in this sample, namely, $3.89 \pm 2 \times 10^{-2} \AA$. This analysis allows for concluding that bright zones in Fig. 5(a) correspond to coherently strained cubic Pm-3m STO, while darker zones in Fig. 5(a) correspond to tetragonal STO. According to Fig. 5(a), the extension of these STO domains is on the order of ten or a few tens of nanometers. They are separated by vertical boundaries, leading to a (quite disturbed) columnarlike morphology. Both STO domains are present in approximately the same proportions in the 10-ML sample, according to Fig. 5(a). Both STO phases are very similar from a chemical point of view so that the difference in the TEM contrast between the STO domains cannot be interpreted as resulting from chemical effects. Tetragonal STO domains are darker because they are slightly misoriented with respect to the Si substrate (mosaicity), opposite to perfectly oriented coherent cubic STO domains. This mosaicity also leads to a quite low contribution of the t-STO phase in the heavily angularly resolved out-of-plane XRD spectra of Fig. 1. 


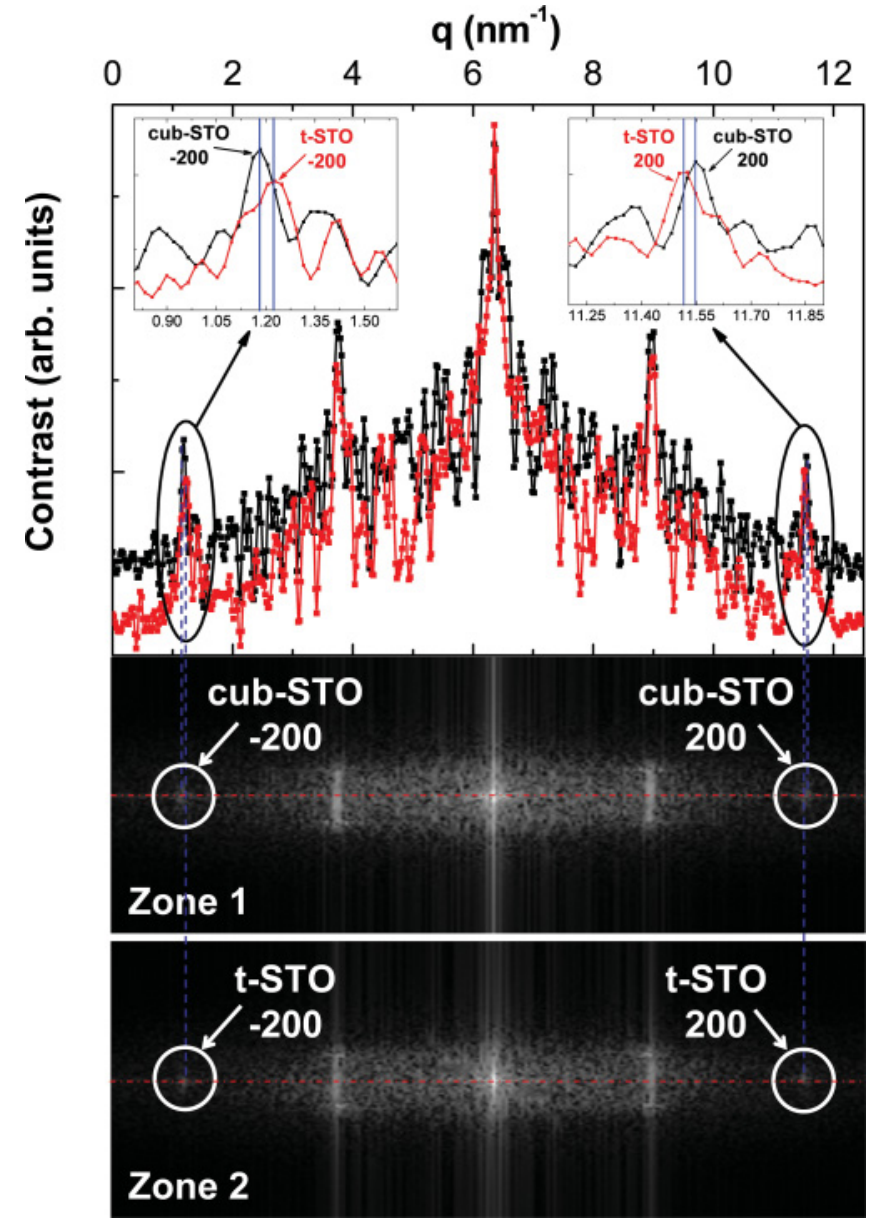

FIG. 6. (Color online) Fourier-transform images of zones 1 and 2 in Fig. 5(a) and associated profiles along the [200] in-plane axis. Insets of the graph: zooms around the STO -200 and STO 200 reflections.

Oppositely, the in-plane experiments summarized in Fig. 2 have been carried out using a much lower angular resolution. In these in-plane spectra, the relative intensities of the peaks corresponding to cubic and tetragonal STO are much more representative of the volume proportion of these domains in the samples.

Thus, the t-STO phase presents a more pronounced mosaicity than the well-ordered coherent cub-STO phase. The coexistence of a well-ordered and a more disordered STO phase appears clearly in the $\omega$ scans of the STO 002 reflection plotted in Fig. 7. Up to $24 \mathrm{ML}$, i.e., as long as the cub-STO phase remains coherently strained on $\mathrm{Si}$, these $\omega$ scans present a sharp central contribution corresponding to a well-ordered STO phase and a much broader contribution corresponding to STO with strong mosaicity. According to the TEM analysis described before, the well-ordered STO phase leading to the sharp central contribution in the $\omega$ scans corresponds to coherently strained cub-STO [bright zone 1 in Fig. 5(a)], while the broader contributions in the $\omega$ scans (due to stronger mosaicity) correspond to the more disordered t-STO phase [dark zone 2 in Fig. 5(a)]. Very similar results as that displayed in Fig. 7 have been published in Ref. 23. In this paper, the authors attribute the sharp portion of the STO $002 \omega$ scans to the presence of coherently strained STO, which is consistent

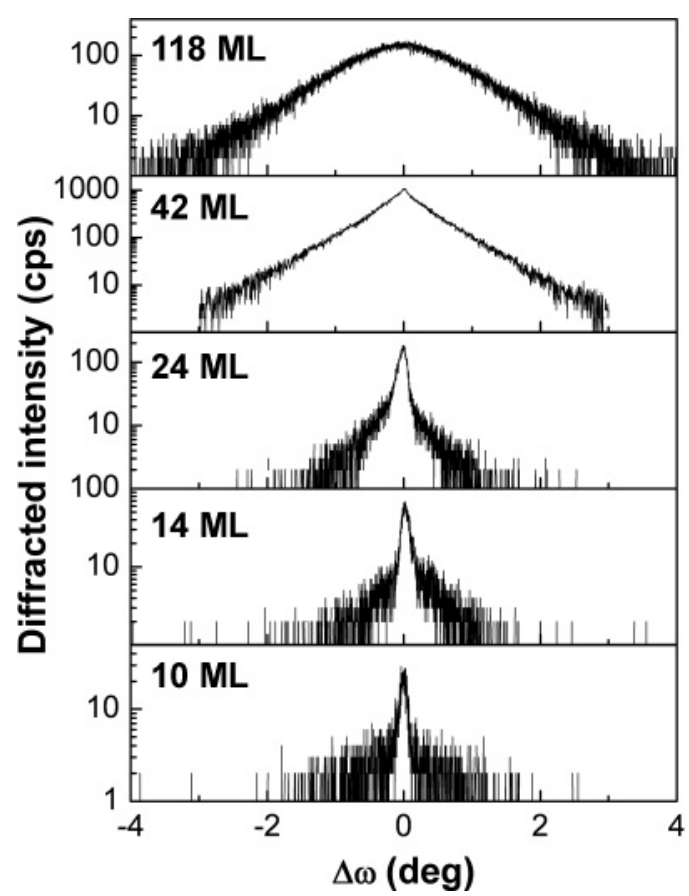

FIG. 7. $\omega$ scans of the STO 002 reflections for 10-, 14-, 24-, 42-, and 118-ML-thick STO/Si layers.

with our analysis. Oppositely, in Ref. 23, the origin of the broader contribution (which is reported by the authors for STO thicknesses as low as $5 \mathrm{ML}$ ) is not clearly discussed and is attributed to a relaxed portion of the STO layer. According to our experimental results, the plastic relaxation critical thickness for the cub-STO/Si systems exceeds $24 \mathrm{ML}$, which excludes the presence of a STO relaxed portion in 5-ML-thick layers. The disordered STO portion in thin layers (up to $24 \mathrm{ML}$ ) corresponds to the t-STO phase, as clearly shown by our experimental results. Above $24 \mathrm{ML}$, plastic relaxation of the cub-STO phase occurs, leading to the disappearance of sharp contribution in the $\omega$ scans of Fig. 7: Plastically relaxed cub-STO presents a strong mosaicity due to the presence of threading defects associated with plastic relaxation.

The evolution of the RHEED pattern at the early stages of STO growth is presented in Fig. 8(a). Pattern (i) corresponds to the $(2 \times 1)$-reconstructed $\mathrm{Si}$ surface before growth, while patterns (ii), (iii), and (iv) were recorded after the growth of $0.8,2$, and $5 \mathrm{ML}$, respectively. The contrast of the diffraction lines in pattern (ii) is low due to the presence of a relatively bright background. This indicates that STO is initially partly amorphous, as mentioned in Sec. I and as described in Ref. 22. The amorphous part of STO then recrystallizes, leading to brighter diffraction line contrast in patterns (ii), (iii), and (iv). The evolution of the RHEED diffraction line contrast and of the STO in-plane lattice parameter a (as deduced from the spacing of the RHEED diffraction lines) is plotted in Fig. 8(b). The diffraction line contrast was measured by normalizing the diffracted intensity with respect to the background intensity (recorded between two diffraction lines). It decreases as compared to that measured for the substrate during the first $\sim 0.8$ STO ML [zone 1 in Fig. 8(a)] and re-increases between 0.8 and $\sim 2 \mathrm{ML}$. Intensity oscillations are then observed, indicating the onset of a two-dimensional layer-by-layer growth mode after 
(a)
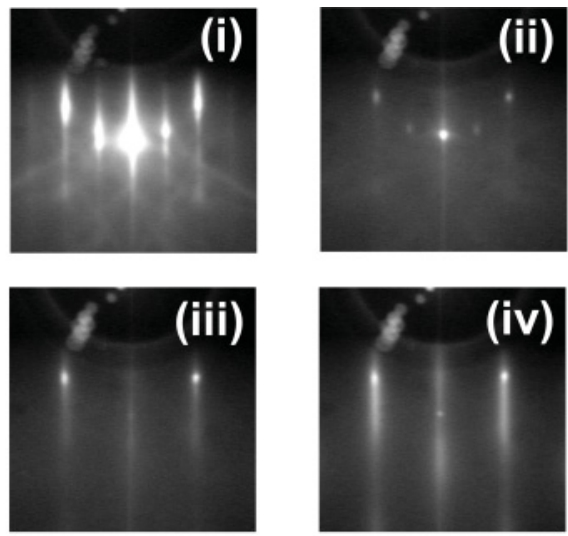

Thickness (ML)

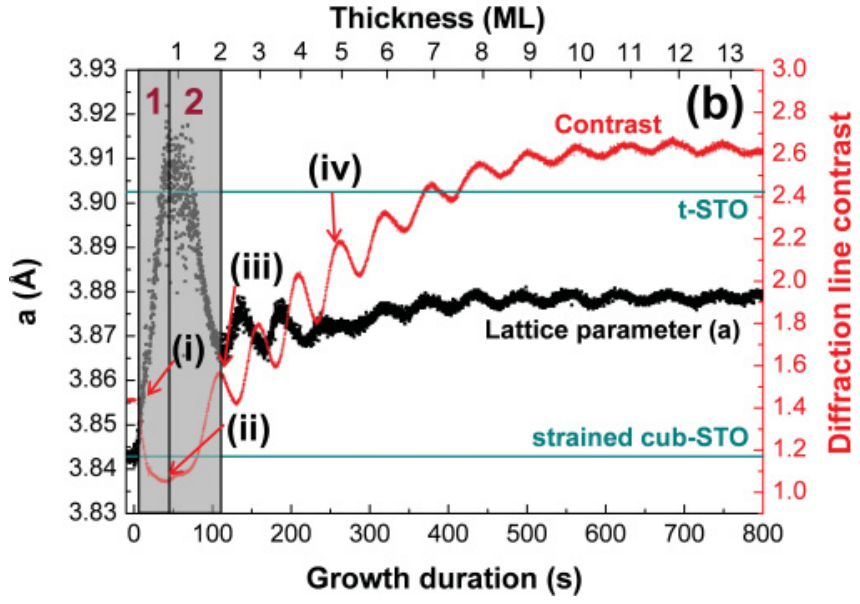

FIG. 8. (Color online) (a) RHEED patterns recorded along the Si [110] azimuth before (i) STO growth, and after (ii) 50, (iii) 110, and (iv) $250 \mathrm{~s}$ of STO growth. (b) (Red circles) Evolution of the RHEED contrast (ratio between the diffraction line intensity and the background intensity) as a function of the deposition time. The points arrowed (i), (ii), (iii), and (iv) correspond to the RHEED patterns of (a). (Black squares) Evolution of the STO in-plane lattice parameter, as deduced from the spacing between STO RHEED diffraction lines. In-plane lattice parameters for fully strained cub-STO and for t-STO at $360{ }^{\circ} \mathrm{C}$, as deduced from XRD and TEM measurements and taking into account the influence of the Si substrate on the thermal expansion of STO during the temperature ramping-down step after growth.

$\sim 2$ ML. The evolution of the RHEED contrast between 0.8 and $2 \mathrm{ML}$ is driven by the proportion of amorphous STO in the growing layer: The RHEED contrast decreases between 0 and 0.8 ML (zone 1) due to the increase of the background intensity and increases above $2 \mathrm{ML}$ (zone 2) due to a decrease of the background intensity. Beyond $2 \mathrm{ML}$, the background intensity remains constant, and the evolution of the RHEED intensity is mostly driven by the variation of the diffraction line intensity. This shows that amorphous STO is formed on the surface simultaneously with crystalline STO up to $0.8 \mathrm{ML}$ and that the amorphous STO part recrystallizes between 0.8 and 2 ML. Interestingly, the evolution of the STO in-plane lattice parameter a is correlated to that of STO crystallinity. Between 0 and $0.8 \mathrm{ML}$, a increases from the Si value $(3.843 \AA$ along the 110 azimuth at the growth temperature) to approximately $3.9 \AA$. This value is very close to that measured by XRD and TEM for the in-plane lattice parameter of the t-STO phase $\left(\sim 3.903 \pm 8 \times 10^{-3} \AA\right.$ at the growth temperature if one considers that the in-plane lattice parameter of t-STO is submitted to the thermal expansion coefficient of Si during the temperature ramp-down after growth). This shows that the initially crystalline STO part is the t-STO phase. Between 0.8 and $2 \mathrm{ML}$, when the amorphous STO part recrystallizes, it starts contributing to RHEED diffraction. In this region (zone 2) the STO in-plane lattice parameter decreases and reaches $\sim 3.865 \AA$ at $2 \mathrm{ML}$. This shows that the in-plane lattice parameter of recrystallized STO is smaller than that of the initially crystalline t-STO phase. Considering the TEM and XRD results detailed earlier, the recrystallized (and initially amorphous) STO portion corresponds to the coherently strained cub-STO phase. Due to its limited resolution, RHEED does not allow discriminating both STO lattice parameters: Beyond 0.8 ML, when both STO phases coexist under their crystalline form in the deposited layer, the lattice parameter measured by RHEED is an average lattice parameter resulting from the contribution of both these phases. Above $2 \mathrm{ML}$, when the initially amorphous phase is fully recrystallized, the surface lattice parameter starts oscillating. These surface lattice parameter oscillations are characteristic for the growth of a coherently strained material. ${ }^{29}$ The simultaneity between lattice parameter oscillation start and full recrystallization of the initially amorphous STO phase further confirms that the initially amorphous STO phase recrystallizes into the coherently strained cub-STO phase between 0.8 and $2 \mathrm{ML}$. The formation of a coherently strained material by recrystallization of its amorphous phase on a substrate may a priori sound anti-intuitive. However, the formation of coherently strained STO on Si by recrystallization of amorphous STO already has been reported earlier by several groups ${ }^{13,14}$ and is a standard technique for fabricating STO on Si.

\section{DISCUSSION ON THE ORIGIN OF THE T-STO PHASE}

At room temperature or even at its growth temperature on $\mathrm{Si}$ $\left(360^{\circ} \mathrm{C}\right)$, the stable bulk STO phase is the cubic $\mathrm{Pm}-3 \mathrm{~m}$ phase. Bulk STO undergoes an extensively studied antiferrodistorsive phase transition at $\sim 105 \mathrm{~K}$ and is ferroelectric with a tetragonal $\mathrm{I} 4 / \mathrm{mcm}$ structure below this temperature. ${ }^{30}$ This phase transition leads to a rotation of the $\mathrm{TiO}_{6}$ octahedrons of the perovskite structure. ${ }^{31}$ It is associated with an increase of the tetragonality (c/a-1) of the STO lattice, which turns from 0 above $105 \mathrm{~K}$ to a few $10^{-4}$ at $50 \mathrm{~K}^{32,33}$ Pressure has been shown to strongly modify the critical temperature of this transition for bulk STO,${ }^{34}$ and strain related to mismatched epitaxial growth is also known as impacting the transition. ${ }^{35-37}$ Thus, a $T_{c}$ as high as $293 \mathrm{~K}$ has been reported for a thin $\mathrm{STO} / \mathrm{DyScO}_{3}$ epitaxial layer. ${ }^{38}$ However, the tetragonality reported in the studies mentioned previously never exceeds $10^{-3}$ and is much smaller than that reported here (between $5 \times 10^{-2}$ and $7 \times 10^{-2}$ for t-STO when the total STO thickness is below $24 \mathrm{ML}$ ).

For the $\mathrm{STO} / \mathrm{Si}(001)$ systems, Aguirre-Tostado et al. ${ }^{25}$ have reported tetragonality values of $1.5 \times 10^{-3}$ for STO thicknesses up to $40 \AA$, while Woicik et al. ${ }^{24}$ have reported a value of $5.6 \times 10^{-2}$ (very close to that reported here) 
for STO thicknesses up to $20 \AA$. In both cases, STO was found to be fully strained on $\mathrm{Si}$, i.e., to present exactly the same in-plane lattice parameter as that of Si. Also, in both cases, the formation of a tetragonal STO phase was attributed to the simultaneous influence of the strain and of an internal polarization related to the presence of interface defects (such as oxygen vacancies) and surface adsorbates. Finally, in both cases, STO was described as single phased.

It is likely that similar effects are at the origin of the formation of the t-STO phase in our samples, even if our experimental results significantly differ from that reported in Refs. 24 and 25. In our samples, grown by direct deposition of partly crystalline STO (as in the low-temperature step of the kinetically limited method), two STO phases coexist. The t-STO phase, while not commensurate with $\mathrm{Si}$ (it presents a larger in-plane lattice parameter than that of $\mathrm{Si}$ ) is crystalline as soon as growth begins while the cub-STO phase results from the spontaneous crystallization of initially amorphous STO. Moreover, the t-STO phase is clearly present for STO thicknesses up to $24 \mathrm{ML}(\sim 96 \AA)$, which is much larger than the maximal thicknesses of 40 and $20 \AA$ reported in Refs. 24 and 25, respectively. In our growth conditions, the amount of oxygen provided at the early stages of the growth is insufficient to fully oxidize the entire material. In this O-poor environment, $\mathrm{O}$ segregation effects (enhanced by the presence of an elevated concentration of $\mathrm{O}$ vacancies) are liable to take place, leading to an inhomogeneous repartition of the $\mathrm{O}$ atoms (and $\mathrm{O}$ vacancies) at the very beginning of the growth that may be at the origin of the formation of the two STO phases. In the end, the fact that the t-STO phase is not coherent with the $\mathrm{Si}$ substrate (which indicates that it is not fully strained on $\mathrm{Si}$ ), while the cub-STO phase is fully strained on $\mathrm{Si}$, indicates that epitaxial strain does not play a major role in the formation of a t-STO phase.

\section{SUMMARY AND CONCLUSIONS}

The study detailed here highlights the complexity of the growth of STO on Si. In our growth conditions, STO is two phased. A cubic STO phase is formed by recrystallization of initially amorphous STO. This STO phase is coherently strained on $\mathrm{Si}$ and obeys elasticity laws (with the elastic constants of bulk cubic Pm-3m STO) up to 24 ML. Above this thickness, plastic relaxation starts leading to fully relaxed STO at $118 \mathrm{ML}$. The tetragonal t-STO phase is crystalline as soon as growth begins and remains tetragonal (with a tetragonality of $5 \times 10^{-2}$ to $6 \times 10^{-2}$ ) up to at least $24 \mathrm{ML}$. Further increase of STO thickness leads to a progressive reduction of the tetragonality of the t-STO phase that tends to 0 for large STO thicknesses. Despite the apparent simultaneity between plastic relaxation of the strained cub-STO phase and diminution of the tetragonality of the t-STO phase (which requires further experimental confirmation by studying STO/Si layers with intermediate thicknesses), epitaxial strain is not likely to be the main driving force for the formation of the t-STO phase: O vacancies associated with $\mathrm{O}$ segregation at the early stages of the growth are more likely at the origin of this phenomenon. Improving the quality of the STO/Si layers and controlling their electrical properties and possible ferroelectric behavior requires a refined control of $\mathrm{O}_{2}$ injection at the early stages of the growth, which is particularly challenging in a MBE environment and may necessitate the development of adequate $\mathrm{O}_{2}$ injection and regulation systems.

\section{ACKNOWLEDGMENTS}

The authors gratefully thank Philippe Regreny and JeanBaptiste Goure for technical assistance. This work was supported by the French Agence Nationale de la Recherche (ANR, Project No. COMPHETI P3N ANR-09-Nano-013-01 and Project No. ANR BLAN-2007 MINOS). *guillaume.saint-girons@ec-lyon.fr

${ }^{1}$ J. Heber, Nature (London) 459, 28 (2009).

${ }^{2}$ A. Ohtomo and H. Y. Hwang, Nature (London) 427, 423 (2004).

${ }^{3}$ P. Zubko and J. M. Triscone, Nature (London) 450, 45 (2009).

${ }^{4}$ V. Garcia, S. Fusil, K. Bouzehouane, S. Enouz-Vedrenne, N. D. Mathur, A. Barthélémy, and M. Bibes, Nature (London) 460, 81 (2009).

${ }^{5}$ M. Gajek, M. Bibes, S. Fusil, K. Bouzehouane, J. Fontcuberta,

A. Barthélémy, and A. Fert, Nat. Mater. 6, 296 (2007).

${ }^{6}$ C. L. Jia, V. Nagarajan, J. Q. He, L. Houben, T. Zhao, R. Ramesh, K. Urban, and R. Waser, Nat. Mater. 6, 64 (2007).

${ }^{7}$ N. Reyren, S. Thiel, A. D. Caviglia, L. Fitting-Kourkoutis, G. Hammerl, C. Richter, C. W. Schneider, T. Kopp, A. S. Rüetschi,

D. Jacquard, M. Gabay, D. A. Müller, J. M. Triscone, and J. Manhart, Science 317, 1196 (2007).

${ }^{8}$ J. F. Scott, Science 315, 954 (2007).

${ }^{9}$ Z. Li, J. Wang, Y. Lin, and C. W. Nan, Appl. Phys. Lett. 96, 162505 (2010).

${ }^{10}$ R. A. McKee, F. J. Walker, and M. F. Chisholm, Phys. Rev. Lett. 81, 3014 (1998).
${ }^{11}$ R. A. McKee, F. J. Walker, and M. F. Chisholm, Science 293, 468 (2001).

${ }^{12}$ X. F. Wang, J. Wang, Q. Li, M. S. Moreno, X. Y. Zhou, J. Y. Dai, Y. Wang, and D. Tang, J. Phys. D: Appl. Phys. 42, 085409 (2009).

${ }^{13}$ H. Li, X. Hu, Y. Wei, Z. Yu, X. Zhang, R. Droopad, A. A. Demkov, J. Edwards, Jr., K. Moore, W. Ooms, J. Kulik, and P. Fejes, J. Appl. Phys. 93, 4521 (2003).

${ }^{14} \mathrm{G}$. Delhaye, C. Merckling, M. El-Kazzi, G. Saint-Girons, M. Gendry, Y. Robach, G. Hollinger, L. Largeau, and G. Patriarche, J. Appl. Phys. 100, 124109 (2006).

${ }^{15}$ S. B. Mi, C. L. Jia, V. Vaithyanathan, L. Houben, J. Schubert, D. G. Schlom, and K. Urban, Appl. Phys. Lett. 93, 101913 (2008).

${ }^{16}$ G. Saint-Girons, P. Regreny, L. Largeau, G. Patriarche, and G. Hollinger, Appl. Phys. Lett. 91, 241912 (2007).

${ }^{17}$ G. Saint-Girons, C. Priester, P. Regreny, G. Patriarche, L. Largeau, V. Favre-Nicolin, G. Xu, Y. Robach, M. Gendry, and G. Hollinger, Appl. Phys. Lett. 92, 241907 (2008).

${ }^{18}$ G. Saint-Girons, J. Cheng, P. Regreny, L. Largeau, G. Patriarche, and G. Hollinger, Phys. Rev. B 80, 155308 (2009). 
${ }^{19}$ Z. Yu, J. Ramdani, J. A. Curless, C. D. Overgaard, J. M. Finer, R. Droopad, K. W. Eisenbeiser, J. A. Hallmark, and W. J. Ooms, J. Vac. Sci. Technol. B 18, 2139 (2000).

${ }^{20}$ X. Hu, H. Li, Y. Liang, Y. Wei, Z. Yu, D. Marshall, J. Edwards Jr., R. Droopad, X. Zhang, A. A. Demkov, and K. Moore, Appl. Phys. Lett. 82, 203 (2003).

${ }^{21}$ G. J. Norga, C. Marchiori, C. Rossel, A. Guiller, J. P. Locquet, H. Siegwart, D. Caimi, J. Fompeyrine, J. W. Seo, and C. Dieker, J. Appl. Phys. 99, 084102 (2006).

${ }^{22}$ G. Niu, G. Saint-Girons, B. Vilquin, G. Delhaye, J. L. Maurice, C. Botella, Y. Robach, and G. Hollinger, Appl. Phys. Lett. 95, 062902 (2009).

${ }^{23}$ M. P. Warusawithana, C. Cen, C. R. Sleasman, J. C. Woicik, Y. Li, L. F. Kourkoutis, J. A. Klug, H. Li, P. Ryan, L. P. Wang, M. Redzyk, D. A. Muller, L. Q. Chen, J. Levy, and D. G. Schlom, Science 324, 367 (2009).

${ }^{24}$ J. C. Woicik, H. Li, P. Zschack, E. Karapetrova, P. Ryan, C. R. Ashman, and C. S. Hellberg, Phys. Rev. B 73, 024112 (2006).

${ }^{25}$ F. S. Aguirre-Tostado, A. Herrera-Gomez, J. C. Woicik, R. Droopad, Z. Yu, D. G. Schlom, P. Zschack, E. Karapetrova, P. Pianetta, and C. S. Hellberg, Phys. Rev. B 70, 201403(R) (2004).

${ }^{26}$ R. O. Bell and G. Rupprecht, Phys. Rev. 129, 90 (1963).

${ }^{27}$ D. R. Lide, CRC Handbook of Chemistry and Physics, 80th ed. (CRC, 2000), p. 1297.
${ }^{28}$ N. Lucas, H. Zabel, H. Morkoç, and H. Unlu, Appl. Phys. Lett. 52, 2117 (1988).

${ }^{29}$ J. Massies and N. Grandjean, Phys. Rev. Lett. 71, 1411 (1993).

${ }^{30}$ P. A. Fleury, J. F. Scott, and J. M. Worlock, Phys. Rev. Lett. 21, 16 (1968).

${ }^{31}$ K. A. Müller, W. Berlinger, and F. Waldner, Phys. Rev. Lett. 21, 814 (1968).

${ }^{32}$ R. Loetzsch, A. Lübke, I. Uschmann, E. Förster, V. Grosse, M. Thuerk, T. Koettig, F. Schmidl, and P. Seidel, Appl. Phys. Lett. 96, 071901 (2010).

${ }^{33}$ L. Cao, E. Sozontov, and J. Zegehagen, Phys. Stat. Sol. A 181, 387 (2000).

${ }^{34}$ M. Guennou, P. Bouvier, J. Kreisel, and D. Machon, Phys. Rev. B 81, 054115 (2010).

${ }^{35}$ A. Antons, J. B. Neaton, K. M. Rabe, and D. Vanderbilt, Phys. Rev. B 71, 024102 (2005).

${ }^{36}$ F. He, B. O. Wells, and S. M. Shapiro, Phys. Rev. Lett. 94, 176101 (2005).

${ }^{37}$ N. A. Pertsev, A. K. Tagantsev, and N. Setter, Phys. Rev. B 61, R825 (2000).

${ }^{38}$ J. H. Haeni, P. Irvin, W. Chang, R. Uecker, P. Reiche, Y. L. Li, S. Choudury, W. Tian, M. E. Hawley, B. Craigo, A. K. Tagantsev, X. Q. Pan, S. K. Streiffer, L. Q. Chen, S. W. Kirschoefer, J. Levy, and D. G. Schlom, Nature (London) 430, 758 (2004). 